\title{
Person-centred care: what nurses can learn from the patient perspective
}

Person-centred health and social care is viewed as fundamental to good care quality in the UK and elsewhere. It has been an aspiration of policy-makers in England for 20 years (DH 1997) and a key concept underpinning pre-registration nurse education standards for as long, as well as a core skills element in the education and training framework for the health and social care workforce (Skills for Health 2017). It seems timely therefore to consider from the perspective of service users, how far this concept has translated into reality in terms of shaping care planning and delivery. National Voices is a coalition of charities that represents people being in control of their health and care; their recent publication (National Voices 2017) brings together evidence concerning the experience of service users of person-centred health and social care in England. Drawing upon data from the Care Quality Commission, the General Practice Patient Survey and the Personal Social Services Adult Social Care Survey amongst others, the National Voices (NV 2017) report highlights some key aspects of service user experiences of person-centred care in practice. What insights can nurses' gain form this to shape education and development needs moving forward?

\section{What is person-centred care?}

The report makes the useful point that whilst there is no single definition of personcentred care (PCC), the term supersedes the more outdated one of patient-centred care which symbolises the dependency that personalisation aims to overcome. For the purpose of the report, PCC was considered under five domains that captured the most common features that this term encompassed: information, communication, involvement in decision-making, care planning and care coordination.

\section{Five domains of PCC and implication for nurse education}

Information: Clearly in order to maximise opportunities for people to take control of their own health care, they need to understand their condition and treatment. NV (2017) reported that whilst $76 \%$ of inpatients felt that their operation and procedure had been completely explained, only one-third received any written information about what to expect post-discharge and only $38 \%$ of those on medications felt they had complete information on side effects. Older people admitted to hospital, many of who have complex comorbidities, are in danger of readmission if insufficient priority is given to advance discharge planning; allocating sufficient time to inform service users and answer questions needs to be role modeled by mentors and skills demonstrators. This client group may have more sensory deficits than other groups and so nurses need to advocate for well-designed and accessible written information particularly around medications.

Communication: personalised and authentic communication is a vital element of feeling valued as a human being. NV (2017) found that whilst $59 \%$ of inpatients believed they always received sufficient emotional support from staff, $24 \%$ felt staff were not always available when they had fears or worries. However in relation to procedures and operations, $83 \%$ felt they had had this explained in such a way they could understand. Communication skills are a key part of the nursing curriculum; active listening is an important facet of this; these findings indicate that this appears to be demonstrated well by staff. Lack of time may account for the negative finding about responding to worries; students need to maximise every contact to make an authentic connection with service users; whatever the task the communicative benefits must to be maximised. 
Involvement in decision-making: If clear information is available and communicated effectively by staff, people should be able to make decisions about their care. $56 \%$ of inpatients felt as involved as they wanted to be in making decisions about treatments. However sometimes this does not translate into practice: for example whilst $75 \%$ of bereaved relatives felt their loved one was involved as much as they wanted to be in decisions about end of life, there appears to be inadequate influence over place of death; $3 \%$ said they would rather die in hospital but yet $49 \%$ actually did so. End of life trajectory is not always easy to predict but nurses can have an advocacy role in ensuring advanced care planning to facilitate effective means to share information between agencies and professions to try to respect place of death decisions.

Care planning: personalised care is supported by care planning involving clients and their families. Interestingly NV (2017) noted that there are no national surveys of hospital patients about care planning involvement. Given the end of life care example above, this gap needs addressed.

Coordination of care: people with long term conditions experience care from many care teams and in many settings but NV (2017) noted that most service users surveys are not designed to capture data on experiences of care co-ordination. Nonetheless $77 \%$ of inpatients were told who to contact if they had worries after hospital discharge but in relation to older people's care although examples of good interagency working are apparent joint working was not mainstream practice, services were duplicated and pressures within different services were poorly understood. From the perspective of nurse education and indeed other health professional education, there is a tendency to focus on one discipline particularly at pre-registration level. Given the growth in integrated care, this may be detrimental to preparing future practitioners adequately for developments in service delivery.

\section{Conclusion}

Many of these findings are of great interest to those involved in pre-registration nurse education both in practice and university settings. A mixed picture emerges of person-centred care from the service user perspective and highlights the benefit of integrating this voice into education delivery.

\section{References}

Department of Health (1997). The New NHS - Modern, Dependable. London: DH)

National Voices (2017). Person-centred Care in 2017. Evidence from Service Users. Available at:

https://www.nationalvoices.org.uk/sites/default/files/public/publications/personcentred care in 2017 - national voices.pdf

Skills for Health (2017). Core Skills Training Framework. Available at: http://www.skillsforhealth.org.uk/services/item/146-core-skills-training-framework

\section{Dr Janet Scammell, Associate Professor (Nursing), Bournemouth University}

